\title{
Predicting serum vitamin D concentrations based on self-reported lifestyle factors and personal attributes
}

\author{
Vikki Ho ${ }^{1,2} \dagger$, Coraline Danieli ${ }^{3} \dagger$, Michal Abrahamowicz ${ }^{3}$, Anne-Sophie Belanger ${ }^{4}$, Vanessa Brunetti ${ }^{1}$, \\ Edgard Delvin ${ }^{5}$, Julie Lacaille ${ }^{1}$ and Anita Koushik ${ }^{1,2 *}$ \\ ${ }^{1}$ Université de Montréal Hospital Research Centre (CRCHUM), 850 Saint-Denis Street, Montreal, QC, Canada H2X OA9 \\ ${ }^{2}$ Department of Social and Preventive Medicine, Université de Montréal, 7101 Avenue du Parc, Montreal, QC, \\ Canada $H 3 N 1 X 9$ \\ ${ }^{3}$ Department of Epidemiology, Biostatistics, and Occupational Health, McGill University, 1020 Pine Avenue West, Montreal, \\ QC, Canada H3A $1 A 2$ \\ ${ }^{4}$ CHU Ste-Justine, 3175 Chemin de la Côte-Sainte-Catherine, Montreal, QC, Canada H3T 1C5 \\ ${ }^{5}$ CHU Ste-Justine Research Centre, Gastroenterology, Hepatology and Nutrition Division, 3175 Chemin de la Côte-Sainte- \\ Catherine, Montreal, QC, Canada H3T 1C5 \\ (Submitted 25 January 2018 - Final revision received 19 May 2018 - Accepted 15 June 2018 - First published online 6 August 2018)
}

\section{Abstract}

Evidence supports the role of vitamin D in various conditions of development and ageing. Serum 25-hydroxyvitamin D (25(OH)D) is the best indicator for current vitamin D status. However, the cost of its measurement can be prohibitive in epidemiological research. We developed and validated multivariable regression models that quantified the relationships between vitamin D determinants, measured through an inperson interview, and serum 25(OH)D concentrations. A total of 200 controls participating in a population-based case-control study in Montreal, Canada, provided a blood specimen and completed an in-person interview on socio-demographic, reproductive, medical and lifestyle characteristics and personal attributes. Serum 25(OH)D concentrations were quantified by liquid chromatography-tandem MS. Multivariable least squares regression was used to build models that predict 25(OH)D concentrations from interview responses. We assessed high-order effects, performed sensitivity analysis using the lasso method and conducted cross-validation of the prediction models. Prediction models were built for users and non-users of vitamin D supplements separately. Among users, alcohol intake, outdoor time, sun protection, dose of supplement use, menopausal status and recent vacation were predictive of $25(\mathrm{OH}) \mathrm{D}$ concentrations. Among non-users, BMI, sun sensitivity, season and recent vacation were predictive of $25(\mathrm{OH}) \mathrm{D}$ concentrations. In cross-validation, $46-47 \%$ of the variation in $25(\mathrm{OH}) \mathrm{D}$ concentrations were explained by these predictors. In the absence of $25(\mathrm{OH}) \mathrm{D}$ measures, our study supports that predicted $25(\mathrm{OH}) \mathrm{D}$ scores may be used to assign exposure in epidemiological studies that examine vitamin $\mathrm{D}$ exposure.

\section{Key words: Vitamin D: Lifestyle: Predictors: Prediction modelling: Cross-validation}

Vitamin D deficiency has recently been described as being pandemic $^{(1)}$, affecting $50 \%$ of the population worldwide ${ }^{(2)}$. Undeniable public health implications may arise as vitamin D insufficiency/deficiency has been associated with various conditions of development and ageing, such as rickets, fractures and osteoporosis, as well as an overall higher risk of all-cause mortality ${ }^{(3,4)}$. Increasingly, the epidemiological evidence is growing to support the role of vitamin $\mathrm{D}$ in the aetiology of chronic diseases like cancer, $\mathrm{CHD}$, diabetes, neurological disorders and so on ${ }^{(2)}$.

The main source of vitamin D is cutaneous exposure to UVB radiation from the sun, which catalyses the conversion of 7-dehydrocholesterol in the skin to vitamin $\mathrm{D}^{(5)}$. However, in
Northern areas such as Canada, UVB irradiation during winter is insufficient for cutaneous production ${ }^{(6)}$. Thus, dietary vitamin D from natural sources (e.g. fish, eggs) and fortified foods (e.g. milk, cereals) and supplements ${ }^{(7)}$ represents an important source. Vitamin D that is endogenously produced or derived from diet is then converted in the liver to 25hydroxycholecalciferol (25-hydroxyvitamin D (25(OH)D)) and finally to $1,25(\mathrm{OH})_{2} \mathrm{D}$ primarily in the kidney ${ }^{(8)}$.

The best available biomarker for total vitamin $\mathrm{D}$ exposure is serum concentration of $25(\mathrm{OH}) \mathrm{D}^{(9)}$. However, the cost of its measurement can be prohibitive in an epidemiological study. A suggested cost-efficient alternative is to use models that

Abbreviations: 25(OH)D, 25-hydroxyvitamin D; NHS, Nurses' Health Study; RMSPE, root mean square prediction error.

* Corresponding author: Dr A. Koushik, email anita.koushik@umontreal.ca

$\dagger$ These authors contributed equally to this work. 
predict serum 25(OH)D from self-reported values of lifestyle and personal attributes associated with $25(\mathrm{OH}) \mathrm{D}^{(10)}$. Such models have been previously developed ${ }^{(10-24)}$ and may have predictive ability for other similar populations. However, models developed within a sub-population of a study, which can then be applied to full study populations, can allow for the consideration of the full range of information that was collected with flexibility on how to best represent the data, which may result in improved prediction ability. We developed and validated multivariable regression models that quantified the relationships between vitamin $\mathrm{D}$ determinants measured through an in-person interview and the concentration of serum $25(\mathrm{OH}) \mathrm{D}$ in a Montreal, Canada, population.

\section{Methods \\ Study population and data collection}

This study was conducted within the PRevention of OVArian Cancer in Quebec (PROVAQ) study, a population-based casecontrol study conducted by our team in Montreal, Canada, from 2011 to $2016^{(25)}$. Cases were women aged 18-79 years with incident epithelial cancer of the ovary, fallopian tube or peritoneum. Population controls, randomly sampled from the Quebec electoral list throughout the recruitment period, were frequency-matched to cases according to 5-year age group and Montreal region. The population for the current study included the first 200 controls that agreed to participate in the PROVAQ study and to provide a blood sample within 2 weeks of participating. This study was conducted according to the guidelines laid down in the Declaration of Helsinki, and all procedures involving human subjects were approved by the Research Ethics Committee of the Centre de recherche du CHUM. Written informed consent was obtained from all participants.

An in-person interview was carried out among all participants to collect information on socio-demographic, reproductive, medical and lifestyle characteristics. Questionnaire items on vitamin D sources included sun exposure during school, work, commuting and leisure activities, as well as sun protection behaviours (e.g. sunscreens, covering body). Dietary vitamin D from natural and fortified foods, supplement use, use of tanning salons and sun vacations was also assessed. Although the questionnaire assessed these exposures over the life course, specific questions also targeted recent exposure (past 2-3 months). The questionnaire also assessed factors related to sun sensitivity, including eye colour, natural hair colour as a teenager, tendency to burn at first summer sun exposure without protection, tanning ability after repeated summer sun exposure and skin tone

\section{Potential predictors}

We considered the following potential predictors based on the most recent exposures occurring in the past 2-3 months (Table 1): age, season of blood draw, self-reported ethnicity, highest education level, menopausal status, BMI, recent sun vacation and sun protection, outdoor time with total sun protection (i.e. arms/legs all covered using clothing or sunscreen), outdoor time with partial sun protection (i.e. arms/legs partially covered using clothing or sunscreen), outdoor time with no sun protection (i.e. no protection from the sun using clothing or sunscreen), vitamin D supplement dose, dietary vitamin D intake, alcohol intake and smoking. Only one woman had recently used tanning salons, and thus this variable could not be considered. Sun vacation was defined as having taken a vacation to a location with a summer climate. Season of blood draw was classified according to summer (April to September) $v$. winter (October to March), based on the months in Canada that have sufficient $v$. insufficient UVB irradiation for cutaneous vitamin D synthesis, respectively ${ }^{(5,26)}$. Outdoor time was based on the accumulated reported time outside for school, work, commuting and leisure activities. Dietary vitamin $\mathrm{D}$ was based on intake of milk, margarine, tuna, salmon (canned, fresh or frozen, smoked), canned sardines and eggs, which are the major natural and fortified dietary sources of vitamin D in Canada ${ }^{(27)}$. The vitamin D content in each food/ beverage, obtained from the Canadian Nutrient File ${ }^{(28)}$, was multiplied by the frequency of intake of a standard serving of that food/beverage and then summed to give total dietary vitamin D intake. We also defined a sun sensitivity score variable based on responses to eye colour, hair colour, skin tone, tendency to burn at first exposure to sun and tanning ability ${ }^{(29)}$. For each of these items, responses were assigned a value between 1 and 4, with low values indicating high sun sensitivity and high values indicating low sensitivity, based on the literature when available ${ }^{(29,30)}$. The sum of the values determined the sun sensitivity score, ranging from 5 (highest sun sensitivity) to 19 (lowest sun sensitivity).

\section{Laboratory analysis}

Serum $25(\mathrm{OH}) \mathrm{D}_{3}, 25(\mathrm{OH}) \mathrm{D}_{2}$ and 3 -epi-25(OH)D 3 concentrations were measured by a liquid chromatography-tandem MS method (Department of Clinical Biochemistry, CHU Ste-Justine $)^{(31,32)}$. Within each batch, two quality control samples, independent of our study population, were included in duplicate. In addition, $10 \%$ of the study population was measured in duplicate in different batches.

Average intra-batch and inter-batch correlations of variation were below 3 and 5\%, respectively. Quantification of serum $25(\mathrm{OH}) \mathrm{D}_{2}, \quad 25(\mathrm{OH}) \mathrm{D}_{3}$ and 3 -epi-25(OH)D 3 concentrations above the limit of detection of our assay was successful for 14 , 199 and 131 participants, respectively. For one participant, ion suppression caused a reduced detector response for $25(\mathrm{OH}) \mathrm{D}_{3}$, $25(\mathrm{OH}) \mathrm{D}_{2}$ and 3 -epi-25(OH)D $\mathrm{D}_{3}$, as well as the internal standards, resulting in uninterpretable values for all three vitamin D metabolites. Concentrations below the limit of detection were assigned a value of 0 . Total serum $25(\mathrm{OH}) \mathrm{D}$ was calculated as the sum of $25(\mathrm{OH}) \mathrm{D}_{2}, 25(\mathrm{OH}) \mathrm{D}_{3}$ and 3 -epi- $25(\mathrm{OH}) \mathrm{D}_{3}$ concentrations (in $\mathrm{nmol} / \mathrm{l}$ ).

\section{Statistical analysis}

We developed a series of model-building steps that are outlined below and described in more detail in the online Supplementary material (Section 1$)$. Seven women were missing values for menopausal status ( $n$ 5) or smoking $(n 2)$; thus, the final sample size was 192. Modelling was conducted using multivariable 
least squares regression, using both the forward and backward selection procedures in SAS 9.3. We also used the lasso method in a sensitivity analysis ${ }^{(33)}$. Whenever the forward and backward procedures yielded different predictors, all predictors selected by at least one of the two procedures were included at the next modelling step. As our initial examination of variable distributions showed that the prevalence of vitamin D supplement use was higher than expected based on past studies ${ }^{(23,34)}$, we conducted a set of preliminary analyses to examine the interaction between each predictor variable and supplement use. We observed several statistically significant interactions (results not shown), and thus we performed all our multivariable model building separately for users ( $n$ 120) and nonusers $(n 72)$ of vitamin D supplements.

Step 1: assessing potential non-linearity. We first assessed whether continuous variables would be better represented with the inclusion of a quadratic term to account for non-linearity. At this step, all categorical variables and the linear terms for continuous variables were forced into the model. Selection of quadratic terms was conducted in one model and limited to the quadratic terms that were statistically significant at an $\alpha$-level of $0 \cdot 15$, which corresponds approximately to the cut-off for improving the model's Akaike information criterion ${ }^{(35)}$.

Step 2: assessing interactions with season. As season is a strong predictor of $25(\mathrm{OH}) \mathrm{D}$ concentrations ${ }^{(11)}$, we hypothesised that season of blood draw may modify relationships between other predictors and $25(\mathrm{OH}) \mathrm{D}$. To ensure that any important interactions with season would be accounted for, we considered the two-way interactions between each of the other predictors and season; all the main effect categorical and linear continuous variables were forced into the model. An $\alpha$-level of 0.15 was used to determine statistical significance of the interaction term.

\section{Step 3: building alternative multivariable 'candidate} models'. After identifying higher-order terms for inclusion in Steps 1 and 2, four alternative multivariable models of different complexity were built.

Model 3.1: main effects only. The model in Step 3.1 was limited to the main effects variables only. Selection was conducted including all categorical variables and linear terms, with no variables forced. An $\alpha$-level of $0 \cdot 15$ was used to select the final model variables.

Model 3.2: main effects, plus quadratic and interaction terms. In Step 3.2, the higher-order terms identified in Steps 1 and 2 were now considered, along with all main effects variables. No variables were forced into the models, and an $\alpha$-level of $0 \cdot 10$ was used to select the final model variables.

Model 3.3: final complex model. In this step, all variables that were selected in Steps 3.1 or 3.2 were considered for selection. No variables were forced into the models, and an $\alpha$-level of 0.05 was used to select the final model variables.
Model 3.4: final simplified model. In this step, we assessed whether the final model in Step 3.3 could be simplified. Thus, the variables considered for selection were those that were selected in Step 3.3 but excluded any higher-order terms. An $\alpha$-level of $0 \cdot 15$ was used to select the final model variables.

Lasso model. In a sensitivity analysis, we estimated prediction models for users and non-users of vitamin D supplements using the lasso method through the 'glmnet' $\mathrm{R}$ package (Section 2, online Supplementary material). All categorical and linear main effects, as well as the higher-order quadratic and interaction terms, were considered simultaneously.

Step 4: validation of model performance. To evaluate the performance of the prediction models generated in Steps $3 \cdot 1$ to 3.4 and the lasso method for users and non-users of vitamin D supplements, we used cross-validation, which approximates the expected performance of the models to predict $25(\mathrm{OH}) \mathrm{D}$ in a future independent data set ${ }^{(36)}$. The performance indicators that we estimated were the $R^{2}$ (percentage of the total variation in observed $25(\mathrm{OH}) \mathrm{D}$ values explained by the model), the Pearson linear correlation coefficient between the predicted and observed $25(\mathrm{OH}) \mathrm{D}$ values and the root mean square prediction error (RMSPE: square root of the mean of the squared differences between the predicted and observed 25(OH)D values). We used the leave-one-out 5 -fold procedure ${ }^{(37)}$, where the original data set (for users and non-users separately) was first randomly partitioned into five equal-size sub-samples (or folds). Subsequently, a random sample of four of the sub-samples was used as a 'training' sample to re-estimate the parameters of a given model, which was then applied to the remaining 'testing' sub-sample to calculate their predicted $25(\mathrm{OH}) \mathrm{D}$ concentrations. The predicted and observed 25(OH)D values were then compared, with the users and non-users pooled, to calculate the aforementioned performance indicators. This was repeated five times such that each of the five sub-samples was used exactly once as a 'testing' sample while each combination of four subsamples was used as a 'training' sample. To obtain stable and unbiased estimates of the performance indicators, we repeated this leave-one-out 5-fold procedure ten times, with different random partitioning of the original data set each time. The performance statistics were then averaged across the fifty iterations (i.e. ten replications of the five folds). To further assess the performance of all final prediction models, we categorised the observed and the predicted 25(OH)D levels into quartiles and quantified the concordance of the two classifications using the area under the receiver operating characteristic curve (AUC) ${ }^{(38)}$.

Application of previously published prediction models. We also evaluated the application of three previously published prediction models $^{(10,11)}$ to our population, one also based on a Canadian population of women in Ontario ${ }^{(11)}$ and the other two based on the Nurses' Health Studies (NHS and NHSII), which represents the largest and most comprehensive modelling endeavour for $25(\mathrm{OH}) \mathrm{D}^{(10)}$. We calculated the predicted $25(\mathrm{OH}) \mathrm{D}$ in our study population using the published regression coefficients for their model variables defined in our population (Section 3, 
Table 1. Distributions of potential vitamin $D$ predictors and their bivariate relationships with total serum 25-hydroxyvitamin $D(25(\mathrm{OH}) \mathrm{D})$ in users and nonusers

of vitamin D supplements

(Mean values and standard deviations and $\beta$-coefficients; numbers and percentages)

\begin{tabular}{|c|c|c|c|c|c|c|c|c|c|c|c|}
\hline \multirow[b]{2}{*}{ Continuous variables } & \multicolumn{6}{|c|}{ Users of vitamin D supplements ( $n$ 120) } & \multicolumn{5}{|c|}{ Non-users of vitamin D supplements ( $n$ 72) } \\
\hline & \multicolumn{2}{|l|}{ Mean } & \multicolumn{2}{|c|}{ SD } & $\beta^{*}$ & $P+$ & Mean & \multicolumn{2}{|c|}{ SD } & $\beta^{*}$ & $P \dagger$ \\
\hline Age at interview (years) & \multicolumn{2}{|l|}{$63 \cdot 2$} & \multicolumn{2}{|c|}{$10 \cdot 3$} & & 0.01 & $55 \cdot 1$ & \multicolumn{2}{|c|}{11.4} & -0.16 & 0.47 \\
\hline BMI $\left(\mathrm{kg} / \mathrm{m}^{2}\right)$ & \multicolumn{2}{|l|}{$25 \cdot 8$} & 5.9 & \multicolumn{2}{|c|}{-0.80} & 0.06 & $27 \cdot 0$ & \multicolumn{2}{|c|}{$7 \cdot 0$} & -0.87 & 0.01 \\
\hline Outdoor time with total sun protection (h/week) & \multicolumn{2}{|l|}{1.3} & 4.8 & \multicolumn{2}{|c|}{0.62} & 0.24 & 2.5 & \multicolumn{2}{|c|}{$5 \cdot 9$} & 0.90 & 0.03 \\
\hline Outdoor time with partial sun protection (h/week) & 11.6 & & $36 \cdot 4$ & & & 0.14 & $18 \cdot 9$ & & & -0.01 & 0.71 \\
\hline Outdoor time with no sun protection (h/week) & 1.1 & & 5.9 & & & 0.60 & $2 \cdot 1$ & & & 0.18 & 0.45 \\
\hline Alcohol intake (g/week) & $61 \cdot 1$ & & $69 \cdot 3$ & & & $<0.01$ & 71.0 & & & 0.01 & 0.69 \\
\hline Smoking (pack-years) & $15 \cdot 4$ & & 28.7 & & & 0.83 & $11 \cdot 7$ & & & -0.25 & 0.06 \\
\hline Supplement vitamin D dose $(\times 100 \mathrm{IU} /$ week $) \ddagger$ & 94.6 & & $75 \cdot 6$ & & & $<0.0001$ & - & & & - & - \\
\hline Dietary vitamin D intake ( $\mu \mathrm{g} /$ week) & 33.7 & & 24.5 & & & 0.70 & 32.1 & & & -0.01 & 0.91 \\
\hline Sun sensitivity score & $13 \cdot 1$ & & $2 \cdot 6$ & & & 0.43 & $13 \cdot 0$ & & & -1.67 & 0.08 \\
\hline Categorical variables & $n$ & $\%$ & & & SD & $P+$ & $n$ & $\%$ & Mean§ & SD & $P+$ \\
\hline Season of blood draw & & & & & & & & & & & \\
\hline $0=$ October-March & 58 & $48 \cdot 3$ & & & 0.13 & 0.31 & 30 & $41 \cdot 7$ & 53.03 & 0.18 & 0.15 \\
\hline $1=$ April-September & 62 & $51 \cdot 7$ & & & 0.18 & & 42 & $58 \cdot 3$ & $60 \cdot 01$ & 0.24 & \\
\hline Ethnicity & & & & & & & & & & & \\
\hline $1=$ French Canadian & 68 & $56 \cdot 6$ & & & 0.12 & 0.06 & 51 & $70 \cdot 8$ & 57.99 & 0.14 & 0.28 \\
\hline $2=$ Non-French Canadian, European & 41 & 34.2 & & & 0.20 & & 14 & $19 \cdot 4$ & $59 \cdot 71$ & 0.30 & \\
\hline $3=$ Other, mixed & 11 & 09.2 & & & 0.32 & & 7 & 9.8 & $45 \cdot 42$ & 0.40 & \\
\hline Education level & & & & & & & & & & & \\
\hline $1=$ high school and less than high school & 52 & $43 \cdot 3$ & & & 0.14 & 0.48 & 18 & $25 \cdot 0$ & 51.79 & 0.24 & 0.21 \\
\hline $2=$ greater than high school & 68 & $56 \cdot 7$ & & & 0.18 & & 54 & $75 \cdot 0$ & 58.87 & 0.27 & \\
\hline Menopausal status & & & & & & & & & & & \\
\hline $0=$ pre-menopausal & 21 & 17.5 & & & 0.22 & $<0.001$ & 33 & $45 \cdot 8$ & $57 \cdot 84$ & 0.17 & 0.78 \\
\hline $1=$ post-menopausal & 99 & 82.5 & & & 0.24 & & 39 & $54 \cdot 2$ & $56 \cdot 48$ & 0.24 & \\
\hline Recent sun vacation and sun protection & & & & & & & & & & & \\
\hline $0=$ no vacation & 103 & $85 \cdot 8$ & & & 0.10 & 0.08 & 55 & $76 \cdot 4$ & $53 \cdot 13$ & 0.13 & $<0.01$ \\
\hline $1=$ vacation with sun protection & 10 & 8.4 & & & 0.33 & & 10 & 13.9 & 74.42 & 0.34 & \\
\hline $2=$ vacation with partial or no sun protection & 7 & 5.8 & & 45 & 0.39 & & 7 & 9.7 & 63.58 & 0.40 & \\
\hline
\end{tabular}

${ }^{*}$ Regression coefficient (slope) from a simple, bivariate linear regression model between variable and 25(OH)D

$\dagger P$-value for the $t$ test of $\mathrm{H}_{0}: \beta=0$ for continuous variables and $\mu=\mu_{0}$ for categorical variables.

$\ddagger$ Mean supplement vitamin $D$ dose is equivalent to 236.5 (SD 199.9) $\mu \mathrm{g} /$ week.

$\S$ Mean of the $25(\mathrm{OH}) \mathrm{D}$ level in the category of interest.

online Supplementary materials), and calculated the Pearson correlation coefficient comparing the predicted to observed $25(\mathrm{OH}) \mathrm{D}$ values.

Justification of sample size. Our sample size was determined by the feasibility and budget limits associated with blood collection and the $25(\mathrm{OH}) \mathrm{D}$ assay. Thus, we can only provide a post hoc assessment of the statistical power ensured by our fixed sample size of 192 (120 users and seventy-two non-users of vitamin D supplements). Power estimation was carried out using the PASS software program (NCSS Statistical Software) ${ }^{(39)}$ for multivariable linear regression. Because candidate predictors included both categorical and continuous variables, and our focus was not on estimating associations with specific exposure variables, we calculated the minimum $R^{2}$ value for an individual predictor variable that can be detected with $80 \%$ power, given the sample size and an $\alpha$-level of 0.05 . In these calculations, we assumed that covariates included in the same multivariable model together explained $20 \%$ of the total variance in $25(\mathrm{OH}) \mathrm{D}$. Under these assumptions, with 120 users of vitamin D supplements, we had $80 \%$ power to detect as significant an individual variable with an $R^{2}$ of $5 \%$, corresponding to a partial correlation of approximately $0 \cdot 22$. For the seventy-two non-users of supplements, we had $80 \%$ power to detect as significant an individual variable with an $R^{2}$ of $8 \%$, corresponding to a partial correlation of about $0 \cdot 28$. If we assumed that covariates included in the same multivariable model together explained more than $20 \%$ of the total variance in $25(\mathrm{OH}) \mathrm{D}$, the minimum detectable $R^{2}$ decreased negligibly. Under the most conservative assumption that covariates do not explain any variance, the detectable $R^{2}$ increased slightly to 6 and $10 \%$ for supplement users and non-users, respectively. Thus, for both models, we had adequate power to identify and, thus, include in the final multivariable model predictors that explained between 5 and $10 \%$ of the total variance in $25(\mathrm{OH}) \mathrm{D}$ concentration.

\section{Results}

\section{Bivariate relationships}

Table 1 summarises the means and distributions of all continuous and categorical potential predictors, respectively, as well as their 
bivariate associations with $25(\mathrm{OH}) \mathrm{D}$, separately for vitamin $\mathrm{D}$ supplement users and non-users. Mean serum 25(OH)D concentrations were higher for vitamin $\mathrm{D}$ supplement users (mean $=91 \cdot 60, \mathrm{sD}=27 \cdot 20)$ compared with non-users $($ mean $=$ $57 \cdot 10, \mathrm{SD}=20 \cdot 44)$. Among vitamin D supplement users, $25(\mathrm{OH}) \mathrm{D}$ concentrations increased with increasing age $(P=0 \cdot 01)$, alcohol intake $(P<0.01)$ and dose of vitamin $\mathrm{D}$ supplement intake $(P<0.0001)$, and decreased with increasing BMI $(P=0 \cdot 06)$. $25(\mathrm{OH}) \mathrm{D}$ concentration was higher for French Canadians than for other ethnicities, as well as for post-menopausal $v$. premenopausal women $(P<0.0001)$ and people who had recently taken a sun vacation $(P=0.08)$ (Table 1$)$. Similar to vitamin $\mathrm{D}$ supplement users, BMI $(P=0 \cdot 01)$ was inversely associated with $25(\mathrm{OH}) \mathrm{D}$ concentration among non-users. In addition, 25(OH)D concentration was positively associated with longer outdoor time with total sun protection $(P=0.03)$, higher in those who had taken a recent sun vacation $(P<0 \cdot 01)$, inversely associated with pack-years of smoking $(P=0.06)$ and inversely associated with sun sensitivity score $(P=0 \cdot 08)$.

\section{Prediction modelling among vitamin D supplement users}

In Step 1, quadratic terms suggesting non-linearity were retained for BMI and vitamin D supplement dose $(P=0.12$ and $P=0 \cdot 07$, respectively). In Step 2 , the interaction between season and menopausal status was retained $(P=0 \cdot 04)$.
Table 2 presents the regression coefficients for the variables retained in each of Steps $3 \cdot 1-3.4$ of model building among vitamin D supplement users. In Step $3 \cdot 1$ that considered all linear and categorical main effects terms only, alcohol, outdoor time with partial sun protection and vitamin D supplement dose were positively associated with 25(OH)D. Ethnicity and menopausal status were also associated with 25(OH)D, with French Canadian and post-menopausal women having higher concentrations. 25(OH)D was higher among those who had recently been on a sun vacation with sun protection as compared with those who did not take a sun vacation.

In Step 3.2, which included the same starting variables as in Step 3.1 along with the higher-order terms identified in Steps 1 and 2, the quadratic terms for BMI and vitamin D supplement dose were retained, as well as the interaction between season and menopausal status. Post-menopausal women had higher $25(\mathrm{OH}) \mathrm{D}$ concentrations than premenopausal women, but the difference was stronger in winter $v$. summer months (Table 2). In addition, the sun sensitivity score was retained in this model and showed that those with a lower sun sensitivity (i.e. high score) had lower $25(\mathrm{OH}) \mathrm{D}$. Associations with alcohol, ethnicity and being on a recent sun vacation were similar to those observed in Step $3 \cdot 1$.

In Step 3.3 where the starting variables included all variables retained in Steps $3 \cdot 1$ and 3.2, all of the same variables as in Step

Table 2. Associations from prediction models of lifestyle factors and personal attributes identified in Steps 3.1 to 3.4 and from the lasso procedure with total serum 25-hydroxyvitamin D $(25(\mathrm{OH}) \mathrm{D})$ for vitamin D supplement users

\begin{tabular}{|c|c|c|c|c|c|c|c|c|c|}
\hline \multirow[b]{2}{*}{ Covariates } & \multicolumn{2}{|c|}{ Step $3 \cdot 1$} & \multicolumn{2}{|c|}{ Step $3 \cdot 2$} & \multicolumn{2}{|c|}{ Step 3.3} & \multicolumn{2}{|c|}{ Step 3.4} & \multirow{2}{*}{$\frac{\text { Lasso }}{\beta \ddagger}$} \\
\hline & $\beta^{*}$ & $P \dagger$ & $\beta^{*}$ & $P \dagger$ & $\beta^{*}$ & $P \dagger$ & $\beta^{*}$ & $P \dagger$ & \\
\hline Intercept & $56 \cdot 65$ & $<0.0001$ & $133 \cdot 12$ & $<0.0001$ & $140 \cdot 41$ & $<0.0001$ & $51 \cdot 87$ & $<0.0001$ & $81 \cdot 67$ \\
\hline Alcohol intake & 0.08 & $<0.01$ & 0.08 & $<0.01$ & 0.08 & $<0.01$ & 0.08 & $<0.01$ & \\
\hline BMI $\left(\mathrm{kg} / \mathrm{m}^{2}\right)$ & - & - & -4.55 & $<0.01$ & -4.54 & 0.01 & & & \\
\hline $\mathrm{BMI}^{2}\left(\mathrm{~kg} / \mathrm{m}^{2}\right)$ & - & - & 0.06 & 0.02 & 0.06 & 0.02 & & & \\
\hline Outdoor time with partial sun protection & 0.13 & 0.02 & 0.10 & 0.06 & $0 \cdot 10$ & 0.06 & 0.12 & 0.03 & \\
\hline Supplement vitamin D dose & $0 \cdot 13$ & $<0.0001$ & 0.39 & 0.01 & 0.13 & $<0.0001$ & $0 \cdot 13$ & $<0.0001$ & 0.049 \\
\hline Supplement vitamin D dose ${ }^{2}$ & - & - & $-1.09 e-03$ & 0.09 & & & & & \\
\hline Sun sensitivity score & - & - & -1.47 & 0.07 & $-1 \cdot 62$ & 0.04 & & & \\
\hline Ethnicity & - & - & & & & & & & \\
\hline French Canadian & Ref. & & Ref. & & & & & & \\
\hline Non-French Canadian, European & -9.74 & 0.03 & -11.03 & $<0.01$ & & & & & \\
\hline Other, mixed & -9.33 & 0.21 & -3.53 & 0.63 & & & & & \\
\hline \multicolumn{10}{|l|}{ Menopausal status } \\
\hline Pre-menopausal & Ref. & & Ref. & & Ref. & & Ref. & & \\
\hline Post-menopausal & 23.71 & $<0.0001$ & $35 \cdot 20$ & $<0.0001$ & 36.58 & $<0.0001$ & 24.62 & $<0.0001$ & $6 \cdot 15$ \\
\hline Season & - & - & & & & & & & \\
\hline October-March & & & Ref. & & Ref. & & & & \\
\hline April-September & & & $18 \cdot 41$ & 0.06 & $14 \cdot 28$ & 0.14 & & & \\
\hline \multicolumn{10}{|l|}{ Season $\times$ menopausal status } \\
\hline $\begin{array}{l}\text { October-March } \times \text { pre-menopausal } \\
\text { April-September } \times \text { post-menopausal }\end{array}$ & - & - & $\begin{array}{l}\text { Ref. } \\
-28.05\end{array}$ & $<0,01$ & $\begin{array}{l}\text { Ref. } \\
-25.04\end{array}$ & ? & & & \\
\hline \multicolumn{10}{|l|}{ Recent sun vacation and sun protection } \\
\hline No vacation & Ref. & & Ref. & & Ref. & & Ref. & & \\
\hline Vacation with sun protection & $20 \cdot 23$ & $<0.01$ & 18.72 & $<0.01$ & 20.09 & $<0.01$ & 21.56 & $<0.01$ & \\
\hline Vacation with partial or no sun protection & -11.97 & 0.17 & -3.95 & 0.64 & -9.48 & 0.26 & -14.97 & 0.09 & \\
\hline
\end{tabular}

Ref., referent values.

* Regression coefficient from the multivariable linear regression model: it corresponds to the slope for continuous covariates and to the average variation in $25(\mathrm{OH}) \mathrm{D}$ level compared with the reference category for categorical variables.

$\dagger P$-value for the $t$ test of $\mathrm{H}_{0}: \beta=0$.

$\ddagger$ Shrinked lasso coefficients. 
3.2 were retained in the model, except for ethnicity and the quadratic term for supplement vitamin D dose (Table 2). In Step 3.4, which started with the final model from Step 3.3 but removed any higher-order terms (i.e. the quadratic term for BMI and the interaction between menopausal status and season), the main BMI effect, the sun sensitivity score and the main season effect were no longer retained.

\section{Prediction modelling among vitamin D supplements non-users}

In Step 1, quadratic terms suggesting non-linearity were retained for BMI, the sun sensitivity score and time spent outdoors without sun protection $(P=0 \cdot 10, P=0 \cdot 13$ and $P=0 \cdot 14$, respectively). In Step 2 , the interaction with season was retained for education level and dietary vitamin $\mathrm{D}$ intake $(P=0.04$ and $P=0 \cdot 14$, respectively).

Table 3 presents the regression coefficients for the variables identified in model building among non-users of vitamin D supplements. In Step 3.1 that considered only linear and categorical main effects terms, age, BMI and the sun sensitivity score were retained and inversely associated with $25(\mathrm{OH}) \mathrm{D}$, whereas higher $25(\mathrm{OH}) \mathrm{D}$ concentrations were observed among postmenopausal $v$. pre-menopausal women, women with a blood draw during the summer $v$. winter season and those having had a recent vacation $v$. not having taken a vacation (Table 3 ).

In Step 3.2 that added the higher-order terms, additional variables that were retained included the quadratic terms for
BMI and the sun sensitivity score, as well as the interaction between education level and season. Step $3 \cdot 3$ that considered all retained terms from Steps $3 \cdot 1$ and 3.2 confirmed the associations with BMI, sun sensitivity score, season, vacation with sun protection and $25(\mathrm{OH}) \mathrm{D}$ (Table 3$)$. The interaction term between educational level and season was not retained. In Step 3.4, which differed from Step 3.3 only by the exclusion of the quadratic terms for BMI and the sun sensitivity score, all retained variables from Step $3 \cdot 3$ were similarly retained (i.e. BMI, sun sensitivity score, season, sun protection on vacation).

\section{Sensitivity analyses using lasso}

The predictors of $25(\mathrm{OH}) \mathrm{D}$ identified by the lasso procedure for users of vitamin D supplements were vitamin D supplement dose and menopausal status, both positively associated with 25(OH)D (Table 2). For non-users of vitamin D supplements, the predictors identified were BMI, for which a nonlinear effect was observed, and recent vacation with sun protection (Table 3).

\section{Performance of the prediction models}

Table 4 presents the cross-validation results for each of the final models identified in Steps 3.1-3.4 and the lasso method. Each predictive model explained a very similar percentage of the total variation in $25(\mathrm{OH}) \mathrm{D}$ concentration, with $R^{2}$ values ranging from 46 to $47 \%$. Accordingly, the Pearson correlation

Table 3. Associations from prediction models of lifestyle factors and personal attributes identified in Steps 3.1 to 3.4 and from the Lasso procedure with total serum 25-hydroxyvitamin $\mathrm{D}(25(\mathrm{OH}) \mathrm{D})$ for non-users of vitamin $\mathrm{D}$ supplements

\begin{tabular}{|c|c|c|c|c|c|c|c|c|c|}
\hline \multirow[b]{2}{*}{ Covariates } & \multicolumn{2}{|c|}{ Step $3 \cdot 1$} & \multicolumn{2}{|c|}{ Step $3 \cdot 2$} & \multicolumn{2}{|c|}{ Step $3 \cdot 3$} & \multicolumn{2}{|c|}{ Step 3.4} & \multirow{2}{*}{$\begin{array}{c}\text { Lasso } \\
\beta \ddagger\end{array}$} \\
\hline & $\beta^{*}$ & $\mathrm{P}+$ & $\beta^{*}$ & $\mathrm{PH}$ & $\beta^{*}$ & $\mathrm{P}+$ & $\beta^{*}$ & $P \dagger$ & \\
\hline Intercept & $122 \cdot 42$ & $<0.0001$ & -6.67 & 0.89 & -8.03 & 0.86 & $102 \cdot 72$ & $<0.0001$ & $58 \cdot 35$ \\
\hline Age (years) & -0.46 & 0.13 & & & & & & & \\
\hline BMI $\left(\mathrm{kg} / \mathrm{m}^{2}\right)$ & -1.06 & $<0.0001$ & 1.80 & 0.25 & 1.61 & 0.30 & -0.95 & $<0.01$ & \\
\hline $\mathrm{BMI}^{2}\left(\mathrm{~kg} / \mathrm{m}^{2}\right)$ & & & -0.04 & 0.08 & -0.04 & 0.09 & & & -0.0038 \\
\hline Sun sensitivity score & $-2 \cdot 12$ & 0.01 & 7.63 & $0 \cdot 18$ & 9.47 & 0.09 & $-2 \cdot 22$ & $<0.01$ & \\
\hline Sun sensitivity score ${ }^{2}$ & & & -0.39 & 0.08 & -0.46 & 0.04 & & & \\
\hline \multicolumn{10}{|l|}{ Education level } \\
\hline High school or less than high school & & & Ref. & & & & & & \\
\hline Greater than high school & & & 9.62 & $0 \cdot 16$ & & & & & \\
\hline \multicolumn{10}{|l|}{ Menopausal status } \\
\hline Pre-menopausal & Ref. & & & & & & & & \\
\hline Post-menopausal & 11.74 & $0 \cdot 10$ & & & & & & & \\
\hline \multicolumn{10}{|l|}{ Season } \\
\hline October-March & Ref. & & Ref. & & Ref. & & Ref. & & \\
\hline April-September & $10 \cdot 06$ & 0.02 & 20.97 & 0.01 & $8 \cdot 70$ & 0.04 & 8.67 & 0.046 & \\
\hline \multicolumn{10}{|l|}{ Season $\times$ education level } \\
\hline October-March $\times$ high school or less than high school & & & Ref. & & & & & & \\
\hline April-September $\times$ greater than high school & & & -16.62 & 0.08 & & & & & \\
\hline \multicolumn{10}{|l|}{ Recent sun vacation and sun protection } \\
\hline No vacation & Ref. & & Ref. & & Ref. & & Ref. & & \\
\hline Vacation with sun protection & 19.46 & 0.002 & $18 \cdot 19$ & $<0.01$ & $18 \cdot 33$ & $<0.01$ & $18 \cdot 75$ & $<0.01$ & 6.01 \\
\hline Vacation with partial or no sun protection & $14 \cdot 18$ & 0.05 & 14.63 & 0.04 & 13.66 & 0.05 & 14.68 & 0.04 & \\
\hline
\end{tabular}

Ref., referent values.

* Regression coefficient from the multivariable linear regression model: it corresponds to the slope for continuous covariates and to the average variation in $25(\mathrm{OH}) \mathrm{D}$ level compared with the reference category for categorical variables.

$\dagger P$-value for the $t$ test of $\mathrm{H}_{0}: \beta=0$.

$\ddagger$ Shrinked lasso coefficients. 
Table 4. Comparison of performance for different models using crossvalidation and AUC statistics

\begin{tabular}{lcccc}
\hline Models $^{*}$ & $R^{2} \dagger$ & Pearson correlation $\ddagger$ & RMSPE§ & AUCII \\
\hline Step 3.1 & 0.47 & 0.71 & 21.43 & 0.80 \\
Step 3.2 & 0.47 & 0.72 & 21.47 & 0.81 \\
Step 3.3 & 0.45 & 0.72 & 21.75 & 0.82 \\
Step 3.4 & 0.46 & 0.71 & 21.44 & 0.80 \\
Lasso & 0.31 & 0.61 & 24.50 & 0.75
\end{tabular}

RMSPE, root mean square prediction error; 25(OH)D, 25-hydroxyvitamin D.

* See Tables 2 and 3 for details of the models built in Steps 3.1-3.4 and from the lasso method.

† The mean percentage of the total variation of the $25(\mathrm{OH}) \mathrm{D}$ values, over ten iterations of the 5-fold cross-validation, explained in the independent 'testing' sub-samples by the respective model, with coefficients estimated from the 'training sub-sample'.

¥ The mean measure of the linear dependence between values predicted by the respective model and the observed values over ten iterations of the 5-fold crossvalidation.

$\S$ The mean root mean squared prediction error, i.e. average absolute difference between the $25(\mathrm{OH}) \mathrm{D}$ values predicted by the respective model and actually observed over ten iterations of the 5 -fold cross-validation.

II Performance of models when 25(OH)D is categorised into quartiles.

coefficients were also very similar across models, and all close to $0 \cdot 7$, indicating a strong correlation between the predicted and observed vitamin D values. Finally, the RMSPE ranged from 21.43 to 21.75 , suggesting that in future applications to similar populations the predicted vitamin $\mathrm{D}$ concentrations based on our models may diverge on average by about $21-22 \mathrm{nmol} / \mathrm{l}$ from the corresponding true values. The model selected by the lasso procedure performed slightly worse, explaining $38 \%$ of the variation of the outcome $\left(R^{2}\right)$, with a Pearson correlation of 0.61 and RMSPE of 24.50

The AUC values indicated satisfactory concordance between quartiles of predicted $25(\mathrm{OH}) \mathrm{D}$, based on each of the four models, and quartiles of actual (measured) 25(OH)D, with the model developed in Step 3.3 having the highest AUC (0.82) and the lasso-based model the lowest AUC (0.75) (Table 4).

\section{Comparison with published prediction models}

When previously published prediction models ${ }^{(10,11)}$ were applied to our population, we found that the models from the NHS by Bertrand et $a l^{(10)}$ and the Ontario study by Sahota et al. ${ }^{(11)}$, respectively, systematically under- and overestimated the concentrations of $25(\mathrm{OH}) \mathrm{D}$ of our participants. The mean difference between the predicted $v$. observed 25 (OH)D values was -50.5 and $-54.7 \mathrm{nmol} / 1$ for the NHS and NHSII models, respectively, by Bertrand et al. ${ }^{(10)}$, and $32 \mathrm{nmol} / 1$ for the models in Sahota et al. ${ }^{(11)}$. The Pearson correlation coefficients between the predicted and observed $25(\mathrm{OH}) \mathrm{D}$ values (which do not depend on the absolute 25 $(\mathrm{OH}) \mathrm{D}$ values) were 0.37 for the Sahota et $a l^{(11)}$ model and 0.39 and 0.14 for the NHS and NHSII models, respectively.

\section{Discussion}

In this study, we developed and validated multivariable models that predict serum concentrations of $25(\mathrm{OH}) \mathrm{D}$, considered the gold-standard measure of current vitamin D status ${ }^{(40-45)}$, from vitamin D-related variables derived from interview responses, as a cost-efficient alternative to biomarkers for measuring vitamin $\mathrm{D}$ in epidemiological studies. We assessed potentially relevant higher-order effects, not usually considered in traditional prediction modelling. In cross-validation analyses, we contrasted more complex models, which incorporated these higher-order terms with simpler models and found that performance was comparable across all the estimated models with an expected $46-47 \%$ of the total variation in $25(\mathrm{OH}) \mathrm{D}$ concentrations explained in an independent sample of women drawn from a population similar to our study participants.

As the performance was so similar across models, our discussion is limited to the simplest model, identified in Step 3.4. Among vitamin D supplement users, predictors of higher $25(\mathrm{OH}) \mathrm{D}$ were increasing alcohol intake, hours spent outside with partial sun protection, dose of vitamin D supplement, being post-menopausal and having taken a recent sun vacation. Among non-users of vitamin D supplements, a lower BMI, higher sun sensitivity, having had their blood drawn in the summer months and having taken a recent sun vacation were associated with higher concentrations of $25(\mathrm{OH}) \mathrm{D}$.

Many previous studies have investigated associations between vitamin D-related variables and serum 25(OH)D concentrations, among which we considered eighteen to be comparable to ours as they considered similar vitamin D predictors in populations residing in regions with similar latitudes ${ }^{(10-24,34,46,47)}$. The predictors most frequently considered among previous studies were season of blood $\operatorname{draw}^{(10-23,34,46,47)}, \mathrm{BMI}^{(10-20,22-24,34,46,47)}$, supplemental vitamin D use $^{(10,11,13-18,21-24,46,47)}$ and age $\mathrm{e}^{(10-15,17,18,20,22-24,34,46,47)}$. As we observed among non-users of vitamin D supplements, a higher BMI has consistently been associated with lower 25(OH)D concentrations in all but one study ${ }^{(16)}$, whereas a summer $v$. winter blood draw has been associated with a higher $25(\mathrm{OH}) \mathrm{D}$ in all studies. Age, however, has been associated with 25(OH)D levels in only eleven of the fourteen studies in which it was considered, and in inconsistent directions ${ }^{(10-15,17,18,20,34,46)}$. Our findings for age were also inconsistent as it was a predictor only among nonusers of vitamin D supplements in model 3.1, and not in other models, nor among vitamin D supplement users. Although cutaneous vitamin D synthesis deteriorates with age, our participants were exposed to vitamin D additionally through dietary and supplemental sources, which may explain the lack of association with age, particularly in supplement users. Regarding vitamin D supplement use, which has been consistently associated with higher $25(\mathrm{OH}) \mathrm{D}$ concentrations in past studies ${ }^{(10-18,21-24,46,47)}$, we did not examine use $v$. non-use as a potential predictor of $25(\mathrm{OH}) \mathrm{D}$ concentrations; rather, we built our models separately for users and non-users given the high prevalence of use in our study population. However, vitamin D supplement dose was considered among users and a positive association with $25(\mathrm{OH}) \mathrm{D}$ concentrations was observed, similar to that seen in eight previous studies that also considered dose $\mathrm{e}^{(10,13,15,16,18,23,24,47)}$

Several studies have also considered outdoor time $\mathrm{e}^{(11-15,17,19-24,34,47)}$ and alcohol intake ${ }^{(10,14,15,18-20,23,47)}$, and increases in each of these variables have been associated with higher 25(OH)D in the majority of studies, consistent with our 
results. Aspects of diet have been considered as predictors of $25(\mathrm{OH}) \mathrm{D}$ in seventeen studies ${ }^{(10-16,18-24,34,46,47)}$, but the majority focused on individual foods or food groups. Only seven studies examined a measure of dietary vitamin D intake from all foods, excluding supplements, as we $\operatorname{did}^{(10,13,16,18-20)}$. Results similar to ours, indicating that dietary vitamin D was not a predictor of $25(\mathrm{OH}) \mathrm{D}$ levels, were observed in most of these studies $^{(13,16,19,20)}$. An absence of a relation between dietary vitamin D intake and 25(OH)D level in multivariable models may reflect a greater importance of other determinants. The intake of dietary vitamin D in our study population was very similar to the general Canadian population ${ }^{(48)}$.

Interestingly, our finding of a positive association with outdoor time was among people who used partial sun protection. In all, eight studies ${ }^{(11,13,15-17,34,46,47)}$ have reported on sun protection use, all of which reported an association with 25 $(\mathrm{OH}) \mathrm{D}$. Our measure of sun protection was combined with outdoor time, which we believe better represents the intensity of sun exposure with outdoor time, as sun protection use reduces cutaneous vitamin $\mathrm{D}$ production ${ }^{(49)}$. In addition, our estimate of outdoor time was not based on a self-reported global estimate by participants, but rather on distinct questions asking participants to report on their patterns of outdoor time occurring during commuting, work and recreation, which would reduce the error in the overall measure of outdoor time. We also observed an association with recent sun vacation, which has only been considered as a predictor in four previous studies $^{(11,12,16,17)}$

Among vitamin D supplement users, we observed that postmenopausal women had higher 25(OH)D levels compared with pre-menopausal women in multivariable models. Only four previous studies ${ }^{(10,11,18,20)}$ have considered the role of menopausal status on $25(\mathrm{OH}) \mathrm{D}$ concentrations, none of which found menopausal status to be a significant predictor of $25(\mathrm{OH}) \mathrm{D}$ in multivariable models. It is not clear why menopausal status would be associated with $25(\mathrm{OH}) \mathrm{D}$ levels, particularly in multivariable models including other vitamin $\mathrm{D}$ predictors. Although vitamin D supplement dose was in our multivariable model, our results pertaining to menopausal status may reflect the fact that all women in this analysis were taking supplements. No other study on predictors of $25(\mathrm{OH}) \mathrm{D}$ has stratified on vitamin D supplement use, as we did.

Our study is the first to consider a sun sensitivity score based on eye colour, hair colour, skin colour, tendency to burn and tanning ability. Among non-users of vitamin D supplements, a lower sun sensitivity was associated with lower 25(OH)D. Other studies have considered elements of sun sensitivity such as skin colour, tendency to burn, tanning ability and constitutive skin pigmentation measures $^{(11,13,14,16,17,20,24,47)}$, among which two studies reported similar findings to ours where a lower sun sensitivity was associated with lower levels of $25(\mathrm{OH}) \mathrm{D}^{(11,24)}$. In general, the variables selected in our models were associated with $25(\mathrm{OH}) \mathrm{D}$ in a manner consistent with what has been previously observed.

In the application of two previously published prediction models $^{(10,11)}$ to our study population, we had all the relevant variables from their models, except UVB flux in the models by Bertrand et al. ${ }^{(10)}$. However, our Montreal population did not have meaningful variability in latitude and altitude. We observed that, based on the correlation coefficient, the published models did not perform as well as our own models performed in our study population, which may suggest that population-specific models may be needed for attaining the best predictive ability. However, it is also possible that our parametrisation of certain variables (e.g. outdoor time combined with sun protection in one variable, separate modelling by vitamin $\mathrm{D}$ supplement use) may have better captured predictors of $25(\mathrm{OH}) \mathrm{D}$

Limitations of this study include the small sample size, which combined with a skewed distribution for some variables may have limited our evaluation of their influence on $25(\mathrm{OH}) \mathrm{D}$ concentrations. Nonetheless, our cross-validation results indicated that our model explained about $45-47 \%$ of the total variation of $25(\mathrm{OH}) \mathrm{D}$ concentrations in an independent sample drawn from our study population. Although the RMSPE of $21-22 \mathrm{nmol} / 1$ is too high to precisely predict $25(\mathrm{OH}) \mathrm{D}$ values for a given individual, as may be needed in a clinical context, we believe that predictions based on our models could be useful in an epidemiological research setting, where the estimation of a relative risk for the association between vitamin $\mathrm{D}$ and a particular disease would be of interest. Indeed, in large population-based epidemiological studies, it may be much easier to collect data on the predictors included in our models than to obtain direct $25(\mathrm{OH}) \mathrm{D}$ measurements. However, the measurement error due to discrepancies between predicted and unknown true 25(OH)D levels will result in reduced statistical power, necessitating larger sample sizes, and relative risk estimates will be attenuated. From this perspective, the fact that we estimated the cross-validated RMSPE is of practical importance for future epidemiological studies that use our prediction model, as this estimate will allow the use of the simulation extrapolation (SIMEX) methodology for measurement error correction $^{(50)}$

In summary, our study found that interview responses to lifestyle and personal attributes were highly predictive of circulating $25(\mathrm{OH}) \mathrm{D}$ in a population of middle-aged women. In the absence of available biomarker measures of vitamin D, results from our study support that predicted 25(OH)D scores derived from interview items may be used to assign exposure in epidemiological studies that aim to examine the relation between vitamin $\mathrm{D}$ and disease risk.

\section{Acknowledgements}

The authors are grateful to France Dumas for carrying out the blood collection, processing and storage. The authors also thank our interviewers Claire Walker, Françoise Pineault and Martine Le Comte.

This work was supported by the Canadian Cancer Society (grant no. 700485). V. H. received a postdoctoral fellowship from the Canadian Institutes of Health Research and Lung Cancer Canada to conduct this work and is currently supported by the Cancer Research Society, Fonds de recherche du Québec - Santé and Ministère de l'Économie, de la Science et de l'Innovation du Québec. M. A. is a James McGill Professor. 
A. K. was supported by the Cancer Research Society-Cancer Guzzo Université de Montréal Award, the Fonds de recherche du Québec - Santé Research Scholar Program and the Canadian Institutes of Health Research New Investigator programme.

Each coauthor has directly participated in the planning, execution or analysis of the study. M. A. and A. K. designed the sub-study within the PROVAQ study; J. L. coordinated recruitment and data collection; A.-S. B. and E. D. carried out the 25(OH)D assays; V. H., C. D., M. A. and A. K. designed the analytic strategy and drafted the manuscript; V. H. and C. D. carried out the data analysis; and V. B. assisted in carrying out the literature review.

The authors declare that there are no conflicts of interest.

\section{Supplementary material}

For supplementary material/s referred to in this article, please visit https://doi.org/10.1017/S000711451800199X

\section{References}

1. Holick MF (2017) The vitamin D deficiency pandemic: approaches for diagnosis, treatment and prevention. Rev Endocr Metab Disord 18, 153-165.

2. Caccamo D, Ricca S, Curro M, et al. (2018) Health risks of hypovitaminosis D: a review of new molecular insights. Int J Mol Sci 19, 892.

3. Gaksch M, Jorde R, Grimnes G, et al. (2017) Vitamin D and mortality: individual participant data meta-analysis of standardized 25-hydroxyvitamin D in 26916 individuals from a European consortium. PLOS ONE 12, e0170791.

4. Pilz S, Grübler M, Gaksch M, et al. (2016) Vitamin D and mortality. Anticancer Res 36, 1379-1387.

5. Hollis BW (2005) Circulating 25-hydroxyvitamin D levels indicative of vitamin D sufficiency: implications for establishing a new effective dietary intake recommendation for vitamin D. J Nutr 135, 317-322.

6. Schwartz GG \& Hanchette CL (2006) UV, latitude, and spatial trends in prostate cancer mortality: all sunlight is not the same (United States). Cancer Causes Control 17, 1091-1101.

7. de Lourdes Samaniego-Vaesken M, Alonso-Aperte E \& VarelaMoreiras G (2012) Vitamin food fortification today. Food Nutr Res 56, 10.3402/fnr.v56i0.5459.

8. Giovannucci E (2005) The epidemiology of vitamin D and cancer incidence and mortality: a review (United States). Cancer Causes Control 16, 83-95.

9. Johnson CS \& Trump DL (2011) Vitamin D and Cancer. New York: Springer.

10. Bertrand KA, Giovannucci E, Liu Y, et al. (2012) Determinants of plasma 25-hydroxyvitamin D and development of prediction models in three US cohorts. Br J Nutr 108, 1889-1896.

11. Sahota H, Barnett H, Lesosky M, et al. (2008) Association of vitamin $\mathrm{D}$ related information from a telephone interview with 25-hydroxyvitamin D. Cancer Epidemiol Biomarkers Prev 17, 232-238.

12. Burnand B, Sloutskis D, Gianoli F, et al. (1992) Serum 25hydroxyvitamin D: distribution and determinants in the Swiss population. Am J Clin Nutr 56, 537-542.

13. Chan J, Jaceldo-Siegl K \& Fraser GE (2010) Determinants of serum 25 hydroxyvitamin D levels in a nationwide cohort of blacks and non-Hispanic whites. Cancer Causes Control 21, 501-511.
14. Cheng TY, Millen AE, Wactawski-Wende J, et al. (2014) Vitamin D intake determines vitamin D status of postmenopausal women, particularly those with limited sun exposure. J Nutr 144, 681-689.

15. Greene-Finestone LS, Berger C, de Groh M, et al. (2011) 25Hydroxyvitamin D in Canadian adults: biological, environmental, and behavioral correlates. Osteoporos Int 22, 1389-1399.

16. Hedlund L, Brembeck P \& Olausson H (2013) Determinants of vitamin D status in fair-skinned women of childbearing age at northern latitudes. PLOS ONE 8, e60864.

17. Richter K, Breitner S, Webb AR, et al. (2014) Influence of external, intrinsic and individual behaviour variables on serum $25(\mathrm{OH}) \mathrm{D}$ in a German survey. J Photochem Photobiol B 140, 120-129.

18. Shirazi L, Almquist M, Malm J, et al. (2013) Determinants of serum levels of vitamin D: a study of life-style, menopausal status, dietary intake, serum calcium, and PTH. BMC Womens Health 13, 33 .

19. Thuesen B, Husemoen L, Fenger M, et al. (2012) Determinants of vitamin D status in a general population of Danish adults. Bone 50, 605-610.

20. Touvier M, Deschasaux M, Montourcy M, et al. (2015) Determinants of vitamin D status in Caucasian adults: influence of sun exposure, dietary intake, sociodemographic, lifestyle, anthropometric, and genetic factors. I Invest Dermatol 135, 378-388.

21. Hypponen E \& Power C (2007) Hypovitaminosis D in British adults at age $45 \mathrm{y}$ : nationwide cohort study of dietary and lifestyle predictors. Am J Clin Nutr 85, 860-868.

22. Hansen JG, Tang W, Hootman KC, et al. (2015) Genetic and environmental factors are associated with serum 25hydroxyvitamin $\mathrm{D}$ concentrations in older African Americans. J Nutr 145, 799-805.

23. Kuhn T, Kaaks R, Teucher B, et al. (2014) Dietary, lifestyle, and genetic determinants of vitamin D status: a cross-sectional analysis from the European Prospective Investigation into Cancer and Nutrition (EPIC)-Germany study. Eur J Nutr $\mathbf{5 3}$, 731-741.

24. Wang W, Ingles SA, Torres-Mejia G, et al. (2014) Genetic variants and non-genetic factors predict circulating vitamin D levels in Hispanic and non-Hispanic White women: the Breast Cancer Health Disparities Study. Int J Mol Epidemiol Genet $\mathbf{5}$, 31-46.

25. Koushik A, Grundy A, Abrahamowicz M, et al. (2017) Hormonal and reproductive factors and the risk of ovarian cancer. Cancer Causes Control 28, 393-403.

26. Webb AR, Kline L \& Holick MF (1988) Influence of season and latitude on the cutaneous synthesis of vitamin $\mathrm{D}_{3}$ : exposure to winter sunlight in Boston and Edmonton will not promote vitamin $\mathrm{D}_{3}$ synthesis in human skin. J Clin Endocrinol Metab 67, 373-378

27. Munasinghe L, Willows N, Yuan Y, et al. (2017) Vitamin D sufficiency of Canadian children did not improve following the 2010 revision of the dietary guidelines that recommend higher intake of vitamin D: an analysis of the Canadian Health Measures Survey. Nutrients $\mathbf{9}, 945$.

28. Government of Canada (2007) Canadian Nutrient File: Health Canada. https://food-nutrition.canada.ca/cnf-fce/index-eng. jsp (accessed July 2017).

29. Tacke J, Dietrich J, Steinebrunner B, et al. (2008) Assessment of a new questionnaire for self-reported sun sensitivity in an occupational skin cancer screening program. BMC Dermatol $\mathbf{8}, 4$.

30. Veierod MB, Weiderpass E, Thorn M, et al. (2003) A prospective study of pigmentation, sun exposure, and risk of cutaneous malignant melanoma in women. J Natl Cancer Inst 95, 1530-1538. 
31. Jensen ME, Ducharme FM, Theoret Y, et al. (2016) Data in support for the measurement of serum 25-hydroxyvitamin D (25OHD) by tandem mass spectrometry. Data Brief $\mathbf{8}$, 925-929.

32. Jensen ME, Ducharme FM, Theoret Y, et al. (2016) Assessing vitamin D nutritional status: is capillary blood adequate? Clin Chim Acta 457, 59-62.

33. Tibshirani R (2011) Regression shrinkage and selection via the lasso: a retrospective. J $R$ Stat Soc Series B Stat Methodol 73 , $273-282$.

34. van der Meer IM, Boeke AJ, Lips P, et al. (2008) Fatty fish and supplements are the greatest modifiable contributors to the serum 25-hydroxyvitamin D concentration in a multiethnic population. Clin Endocrinol (Oxf) 68, 466-472.

35. Steyerberg EW, Eijkemans MJ, Harrell FE Jr, et al. (2000) Prognostic modelling with logistic regression analysis: a comparison of selection and estimation methods in small data sets. Stat Med 19, 1059-1079.

36. Harrell FE (2015) Regression Modeling Strategies. Springer Series in Statistics. Cham, Switzerland: Springer.

37. Stone M (1974) Cross-validatory choice and assessment of statistical predictions. J R Stat Soc Series B Stat Methodol 36, 111-147.

38. Hand DJ \& Till RJ (2001) A simple generalisation of the area under the ROC curve for multiple class classification problems. Machine Learning 45, 171-186.

39. NCSS Statistical Software (2008) PASS Sample Size. Kaysville, UT: NCSS, LCC.

40. Horst R, Reinhardt T \& Reddy G (2005) Vitamin D metabolism. In Vitamin D, 2nd ed., pp. 15-36 [D Feldman, J Pike and F Glorieux, editors]. New York: Elsevier Academic Press.
41. Cashman KD (2007) Calcium and vitamin D. Novartis Found Symp 282, 123-138; discussion 138-142, 212-128.

42. Holick MF, MacLaughlin JA, Clark MB, et al. (1980) Photosynthesis of previtamin $\mathrm{D}_{3}$ in human skin and the physiologic consequences. Science 210, 203-205.

43. Holick MF, MacLaughlin JA \& Doppelt SH (1981) Regulation of cutaneous previtamin $\mathrm{D}_{3}$ photosynthesis in man: skin pigment is not an essential regulator. Science $\mathbf{2 1 1}$, 590-593.

44. Lehmann B (2005) The vitamin $\mathrm{D}_{3}$ pathway in human skin and its role for regulation of biological processes. Photochem Photobiol 81, 1246-1251.

45. Webb AR (2006) Who, what, where and when - influences on cutaneous vitamin D synthesis. Prog Biophys Mol Biol 92, $17-25$.

46. Looker AC, Pfeiffer CM, Lacher DA, et al. (2008) Serum 25hydroxyvitamin D status of the US population: 1988-1994 compared with 2000-2004. Am J Clin Nutr 88, 1519-1527.

47. Knight JA, Wong J, Cole DEC, et al. (2017) Predictors of 25-hydroxyvitamin D concentration measured at multiple time points in a multiethnic population. Am J Epidemiol 186, $1180-1193$

48. Statistics Canada (2004) Canadian Community Health Survey, Cycle 2.2, Nutrition. Ottawa, ON: Statistics Canada.

49. Libon F, Courtois J, Le Goff C, et al. (2017) Sunscreens block cutaneous vitamin D production with only a minimal effect on circulating 25-hydroxyvitamin D. Arch Osteoporos 12, 66.

50. Cook JR \& Stefanski LA (1994) Simulation-extrapolation estimation in parametric measurement error models. $J \mathrm{Am}$ Stat Assoc 89, 1314-1328. 